\title{
HERMITE-HADAMARD INEQUALITIES FOR UNIFORMLY CONVEX FUNCTIONS AND ITS APPLICATIONS IN MEANS
}

\author{
H. BARSAM AND A. R. SATTARZADEH
}

This paper is dedicated to Professor Hossien Mohebi on his $60^{\text {th }}$ birthday.

Received 15 June, 2019

\begin{abstract}
In this paper, we prove Hermite-Hadamard inequality for uniformly convex, uniformly s-convex functions. Also, we obtain Hermite Hadamard inequality for fractional integral by using these functions. Finally, some applications of these inequalities are given.
\end{abstract}

2010 Mathematics Subject Classification: 26D15; 26D07; 39B62

Keywords: Hermite-Hadamard inequality, uniformly convex, uniformly s-convex

\section{INTRODUCTION AND PRELIMINARIES}

Let $f: I \subset \mathbb{R} \rightarrow \mathbb{R}$ be a convex function and $a, b \in I$ with $a<b$, then the following inequality holds:

$$
f\left(\frac{a+b}{2}\right) \leq \frac{1}{b-a} \int_{a}^{b} f(x) d x \leq \frac{f(a)+f(b)}{2} .
$$

The above inequality is well known in the literature as the Hermite-Hadamard inequality. Recently, the generalizations, improvements, variations and applications for convexity and the Hermite-Hadamard inequality have attracted the attention of many researchers, see $[4-8,11]$ and the references therein.

The following definitions can be found in $[2,12]$ and [1].

Definition 1. Let $f: \mathbb{R} \rightarrow \mathbb{R}$ be a function. Then $f$ is called uniformly convex with modulus $\psi:[0,+\infty) \rightarrow[0,+\infty]$ if $\psi$ is incresaing, $\psi$ vanishes only at 0 , and

$$
f(t x+(1-t) y)+t(1-t) \psi(|x-y|) \leq t f(x)+(1-t) f(y),
$$

for each $x, y \in \mathbb{R}$ and $t \in(0,1)$.

If (1.1) holds with $\psi=\frac{\beta}{2}|.|^{2}$ for some $\beta>0$, then $f$ is called strongly convex with constant $\beta$.

In the following we give a simple example of a uniformly convex function (see [2], Corollary 2.14). 
Example 1. In view of the following equality,

$$
(\alpha x+(1-\alpha) y)^{2}+\alpha(1-\alpha)(x-y)^{2}=\alpha x^{2}+(1-\alpha) y^{2},
$$

for all $\alpha \in(0,1)$ and $x, y \in \mathbb{R}$, the function $f(t)=t^{2}$ for $t \in \mathbb{R}$ is uniformly convex with modulus $\psi(t)=t^{2}$ for all $t \geq 0$.

In the following proposition, the relation between convex functions and strongly convex functions is expressed. For more details about uniformly and strongly convex functions see [2].

Proposition 1. Let $f: \mathbb{R} \rightarrow \mathbb{R}$ be a function and $\beta>0$. Then $f$ is a strongly convex function with constant $\beta$ if and only if $f-\frac{\beta}{2}||^{2}$ is a convex function.

Clearly, strong convexity implies uniformly convexity, uniformly convexity implies strict convexity, and strict convexity implies convexity.

We can define the concept of uniformly s-convexity as follows:

Definition 2. Let $f: \mathbb{R} \rightarrow \mathbb{R}$ be a function. Then $f$ is called s-uniformly convex function with modulus $\psi:[0,+\infty) \rightarrow[0,+\infty]$ if $\psi$ is incresaing, $\psi$ vanishes only at 0 , and

$$
f(t x+(1-t) y)+t^{s}(1-t) \psi(|x-y|) \leq t^{s} f(x)+(1-t)^{s} f(y),
$$

for each $x, y \in \mathbb{R}, t \in(0,1)$ and $s \in(0,1)$.

If Definition (1.2) holds with $\psi=\frac{\beta}{2}|\cdot|^{2}$ for some $\beta>0$, then $f$ is called strongly s-convex with constant $\beta$.

Definition 3. Let $f \in L[a, b]$. The left-sided and right-sided Riemann-Liouville fractional integrals $J_{a^{+}}^{\alpha} f$ and $J_{b^{-}}^{\alpha} f$ of order $\alpha>0$ with $a \geq 0$ are defined by

$$
\begin{aligned}
& J_{a^{+}}^{\alpha} f(x)=\frac{1}{\Gamma(\alpha)} \int_{a}^{x}(x-t)^{\alpha-1} f(t) d t \text { with } x>a \\
& J_{b^{-}}^{\alpha} f(x)=\frac{1}{\Gamma(\alpha)} \int_{x}^{b}(t-x)^{\alpha-1} f(t) d t \text { with } x<b
\end{aligned}
$$

respectively, where $\Gamma(\alpha)$ is the Gamma function and its defnition is

$$
\Gamma(\alpha)=\int_{0}^{+\infty} e^{-t} t^{\alpha-1} d t .
$$

It is to be noted that $J_{a^{+}}^{0} f(x)=J_{b^{-}}^{0} f(x)=f(x)$. In the case of $\alpha=1$, the fractional integral reduces to the classical integral.

In [12], M. Z. Sarikaya et al. presented the following Hermite-Hadamard's inequalities for fractional integrals. 
Theorem 1 ([12]). Let $f: I \rightarrow \mathbb{R}$ be a positive function with $0 \leq a<b$ and $f \in$ $L[a, b]$. If $f$ is a convex function on $[a, b]$, then the following inequality for fractional integrals holds:

$$
f\left(\frac{a+b}{2}\right) \leq \frac{\Gamma(\alpha+1)}{2(b-a)^{\alpha}}\left[J_{a^{+}}^{\alpha} f(b)+J_{b^{-}}^{\alpha} f(a)\right] \leq \frac{f(a)+f(b)}{2} .
$$

\section{MAIN RESUltS}

In this section, we shall state our main results. At the first, we obtain HermiteHadamard type inequalities for the class of uniformly convex, uniformly s-convex and strongly convex functions.

Theorem 2. Let $f: \mathbb{R} \rightarrow \mathbb{R}$ be uniformly convex function. Then, the following inquality holds:

$f\left(\frac{a+b}{2}\right)+\frac{1}{8(b-a)} \int_{a-b}^{b-a} \psi(|t|) d t \leq \frac{1}{b-a} \int_{a}^{b} f(t) d t \leq \frac{f(a)+f(b)}{2}-\frac{1}{6} \psi(|a-b|)$.

Proof. In (1.1), set $t=\frac{1}{2}$, then one has

$$
f\left(\frac{x+y}{2}\right)+\frac{1}{4} \psi(|x-y|) \leq \frac{f(x)+f(y)}{2} .
$$

Now in (2.1), set $x=t a+(1-t) b$ and $y=(1-t) a+t b$, and integrate inequality (2.1) on $[0,1]$ with respect to $t$. We conclude

$$
\begin{aligned}
f\left(\frac{a+b}{2}\right)+\frac{1}{4} \int_{0}^{1} \psi(|(2 t-1)(a-b)|) d t & \\
& \leq \frac{1}{2} \int_{0}^{1} f(t a+(1-t) b) d t+\frac{1}{2} \int_{0}^{1} f((1-t) a+t b) d t .
\end{aligned}
$$

Also, the following equalities holds

$$
\begin{aligned}
\frac{1}{4} \int_{0}^{1} \psi(|(2 t-1)(a-b)|) d t & =\frac{1}{4} \int_{b-a}^{a-b} \psi(|u|) \frac{d u}{2(a-b)} \\
& =\frac{1}{8(b-a)} \int_{a-b}^{b-a} \psi(|t|) d t
\end{aligned}
$$

and

$$
\int_{0}^{1} f((1-t) a+t b) d t=\int_{0}^{1} f(t a+(1-t) b) d t=\frac{1}{b-a} \int_{a}^{b} f(t) d t .
$$

Therefore,

$$
f\left(\frac{a+b}{2}\right)+\frac{1}{8(b-a)} \int_{a-b}^{b-a} \psi(|t|) d t \leq \frac{1}{b-a} \int_{a}^{b} f(t) d t
$$


On the other hand, in (1.1) put $x=a, y=b$ and integrate on $[0,1]$ with respect to $t$. Hence

$$
\int_{0}^{1} f(t a+(1-t) b) d t+\int_{0}^{1} t(1-t) \psi(|a-b|) d t \leq \int_{0}^{1} \frac{f(a)+f(b)}{2} d t,
$$

and so

$$
\frac{1}{b-a} \int_{a}^{b} f(t) d t+\psi(|a-b|) \frac{\Gamma(2) \Gamma(2)}{\Gamma(4)} \leq \frac{f(a)+f(b)}{2} .
$$

Therefore,

$$
\frac{1}{b-a} \int_{a}^{b} f(t) d t \leq \frac{f(a)+f(b)}{2}-\frac{1}{6} \psi(|a-b|),
$$

which completes the proof. It is worth noting that we used the following fact:

$$
\int_{0}^{1} t(1-t) d t=B(2,2)=\frac{\Gamma(2) \Gamma(2)}{\Gamma(4)}=\frac{1}{6},
$$

where

$$
\begin{aligned}
& B(x, y)=\int_{0}^{1} t^{x-1}(1-t)^{y-1} d t, \Gamma(x)=\int_{0}^{+\infty} e^{-t} t^{x-1} d t, x>0, y>0, \\
& B(x, y)=\frac{\Gamma(x) \Gamma(y)}{\Gamma(x+y)} .
\end{aligned}
$$

In order to prove the main theorems, we need the following lemma that has been proved in [3].

Lemma 1. Let $f: I^{o} \rightarrow \mathbb{R}$ be a differentiable function on $I^{o}, a, b \in I^{o}$ with $a<b$. If $f^{\prime} \in L[a, b]$, then the following equality holds:

$$
\frac{f(a)+f(b)}{2}-\frac{1}{b-a} \int_{a}^{b} f(t) d t=\frac{b-a}{2} \int_{0}^{1}(1-2 t) f^{\prime}(t a+(1-t) b) d t .
$$

Theorem 3. Let $f: I^{o} \rightarrow \mathbb{R}$ be a differentiable function on $I^{o}, a, b \in I^{o}$ with $a<b$. If $\left|f^{\prime}\right|$ is uniformly convex function on $I^{o}$, then the following inequality holds:

$$
\left|\frac{f(a)+f(b)}{2}-\frac{1}{b-a} \int_{a}^{b} f(t) d t\right| \leq \frac{b-a}{8}\left(\left|f^{\prime}(a)\right|+\left|f^{\prime}(b)\right|\right)-\frac{b-a}{32} \psi(|a-b|) .
$$

Proof. In view of Lemma 1 and uniformly convexity of $\left|f^{\prime}\right|$, one has

$$
\begin{aligned}
& \left|\frac{f(a)+f(b)}{2}-\frac{1}{b-a} \int_{a}^{b} f(t) d t\right| \leq \frac{b-a}{2} \int_{0}^{1}|(1-2 t)|\left|f^{\prime}(t a+(1-t) b)\right| d t \\
& \leq \frac{b-a}{2} \int_{0}^{1}|1-2 t|\left(t\left|f^{\prime}(a)\right|+(1-t)\left|f^{\prime}(b)\right|+t(t-1) \psi(|a-b|)\right) d t \\
& \leq \frac{b-a}{2} \int_{0}^{1} t|1-2 t|\left|f^{\prime}(a)\right| d t+\int_{0}^{1}|1-2 t|(1-t)\left|f^{\prime}(b)\right| d t
\end{aligned}
$$




$$
\begin{aligned}
& \left.+\int_{0}^{1}|1-2 t| t(t-1) \psi(|a-b|)\right) d t \\
\leq & \frac{b-a}{8}\left(\left|f^{\prime}(a)\right|+\left(f^{\prime}(b)\right)\right)-\frac{b-a}{32} \psi(|a-b|),
\end{aligned}
$$

which completes the proof. Also, note that

$$
\begin{aligned}
& \int_{0}^{1} t|1-2 t| d t=\int_{0}^{1}(1-t)|1-2 t| d t=\frac{1}{4} \\
& \int_{0}^{1}|1-2 t| t(t-1) \psi(|a-b|) d t=-\frac{1}{16} \psi(|a-b|) .
\end{aligned}
$$

Theorem 4. Let $f: I^{o} \rightarrow \mathbb{R}$ be a differentiable mapping on $I^{o}, a, b \in I^{o}$ with $a<b$ and $p>1$. If $\left|f^{\prime}\right|^{q}$ is uniformly convex on $I^{o}$, then the following inequality holds:

$$
\left.\left|\frac{f(a)+f(b)}{2}-\frac{1}{b-a} \int_{a}^{b} f(t) d t\right| \leq \frac{b-a}{2(p+1)^{\frac{1}{p}}} \frac{\left|f^{\prime}(a)\right|^{q}+\left|f^{\prime}(b)\right|^{q}}{2}-\frac{1}{6} \psi(|a-b|)\right)^{\frac{1}{q}},
$$

where $\frac{1}{p}+\frac{1}{q}=1$.

Proof. By Lemma 1 and Hölder's inequality, we conclude

$$
\begin{aligned}
& \left|\frac{f(a)+f(b)}{2}-\frac{1}{b-a} \int_{a}^{b} f(t) d t\right| \leq \frac{b-a}{2} \int_{0}^{1}|(1-2 t)|\left|f^{\prime}(t a+(1-t) b)\right| d t \\
& \leq \frac{b-a}{2}\left(\int_{0}^{1}|1-2 t|^{p} d t\right)^{\frac{1}{p}}\left(\int_{0}^{1}\left|f^{\prime}(t a+(1-t) b)\right|^{q} d t\right)^{\frac{1}{q}} \\
& \leq \frac{b-a}{2} \frac{1}{(p+1)^{\frac{1}{p}}}\left(|f(a)|^{q} \int_{0}^{1} t d t+\left|f^{\prime}(b)\right|^{q} \int_{0}^{1}(1-t) d t+\psi(|a-b|) \int_{0}^{1} t(t-1) d t\right)^{\frac{1}{q}} \\
& \leq \frac{b-a}{2(p+1)^{\frac{1}{p}}}\left(\frac{\left|f^{\prime}(a)\right|^{q}+\left|f^{\prime}(b)\right|^{q}}{2}-\frac{1}{6} \psi(|a-b|)\right)^{\frac{1}{q}} .
\end{aligned}
$$

Hence, the proof is complete.

Theorem 5. Let $f: \mathbb{R} \rightarrow \mathbb{R}$ be strongly convex function. Then

$$
f\left(\frac{a+b}{2}\right)+\frac{\beta}{24}(b-a)^{2} \leq \frac{1}{b-a} \int_{a}^{b} f(t) d t \leq \frac{f(a)+f(b)}{2}-\frac{\beta}{12}(b-a)^{2} .
$$

Proof. From Hermite-Hadamard inequality for convex functions, we have

$$
f\left(\frac{a+b}{2}\right) \leq \frac{1}{b-a} \int_{a}^{b} f(t) d t \leq \frac{f(a)+f(b)}{2} .
$$

Since from Proposition $1 f$ is a strongly convex function, we have $f-\frac{\beta}{2}|\cdot|^{2}$ is convex. Hence in (2.2) replace $f$ by $f-\frac{\beta}{2}|.|^{2}$ and after some calculations the result is obtained. 
Theorem 6. Let $f: \mathbb{R} \rightarrow \mathbb{R}$ be uniformly s-convex function. Then

$$
\begin{aligned}
2^{s-1} f\left(\frac{a+b}{2}\right)+\frac{1}{8(b-a)} \int_{a-b}^{b-a} \psi(|t|) d t & \leq \frac{1}{b-a} \int_{a}^{b} f(t) d t \\
& \leq \frac{f(a)+f(b)}{s+1}-\frac{1}{(s+1)(s+2)} \psi(|a-b|) .
\end{aligned}
$$

Proof. In (1.2), set $t=\frac{1}{2}$, then we have

$$
f\left(\frac{x+y}{2}\right)+\frac{1}{2^{s+1}} \psi(|x-y|) \leq \frac{f(x)+f(y)}{2^{s}} .
$$

Now, set $x=t a+(1-t) b$ and $y=(1-t) a+t b$ in (2.5) and integrate on $[0,1]$ with respect to $t$. We get

$$
\begin{aligned}
f\left(\frac{a+b}{2}\right)+\frac{1}{2^{s+1}} \int_{0}^{1} \psi(\mid & (2 t-1)(a-b) \mid) d t \\
& \leq \frac{1}{2^{s}} \int_{0}^{1} f(t a+(1-t) b) d t+\frac{1}{2^{s}} \int_{0}^{1} f((1-t) a+t b) d t .
\end{aligned}
$$

Now,

$$
\begin{aligned}
\frac{1}{2^{s+1}} \int_{0}^{1} \psi(|(2 t-1)(a-b)|) d t & =\frac{1}{2^{s+1}} \int_{b-a}^{a-b} \psi(|u|) \frac{d u}{2(a-b)} \\
& =\frac{1}{2^{s+2}(b-a)} \int_{a-b}^{b-a} \psi(|t|) d t .
\end{aligned}
$$

Also, we have $\int_{0}^{1} f((1-t) a+t b) d t=\int_{0}^{1} f((1-t) b+t a) d t=\frac{1}{b-a} \int_{a}^{b} f(t) d t$. Therefore

$$
f\left(\frac{a+b}{2}\right)+\frac{1}{2^{s+2}(b-a)} \int_{a-b}^{b-a} \psi(|t|) d t \leq \frac{1}{2^{s-1}(b-a)} \int_{a}^{b} f(t) d t .
$$

On the other hand, in (1.1) put $x=a, y=b$ and integrate on $[0,1]$ with respect to $t$. Then we obtain

$$
\int_{0}^{1} f(t a+(1-t) b) d t+\int_{0}^{1} t^{s}(1-t) \psi(|a-b|) d t \leq \int_{0}^{1} t^{s} f(a)+(1-t)^{s} f(b) d t
$$
so,

$$
\frac{1}{b-a} \int_{a}^{b} f(t) d t+\psi(|a-b|) \frac{\Gamma(s+1) \Gamma(2)}{\Gamma(s+3)} \leq \frac{f(a)+f(b)}{s+1}
$$

finally,

$$
\frac{1}{b-a} \int_{a}^{b} f(t) d t \leq \frac{f(a)+f(b)}{s+1}-\frac{1}{(s+1)(s+2)} \psi(|a-b|),
$$

which completes the proof. 
Theorem 7. Let $p \in[2,+\infty)$, then the following inequality holds:

$$
\begin{aligned}
& \left|\frac{a+b}{2}\right|^{p}+\frac{1}{8(b-a)} 2^{1-p} \min \left\{p 2^{-\frac{p}{2}}, 1-2^{-\frac{p}{2}}\right\} \int_{a-b}^{b-a}|t|^{p} d t \leq \frac{1}{b-a} \int_{a}^{b}|t|^{p} d t \\
& \leq \frac{|a|^{p}+|b|^{p}}{2}-\frac{1}{6} \min \left\{p 2^{-\frac{p}{2}}, 1-2^{-\frac{p}{2}}\right\}|a-b|^{p} .
\end{aligned}
$$

Proof. According to ([2], Proposition 10.13), since $|.|^{2}$ is uniformly convex with modules of convexity $|.|^{2}$. Hence for $p \in[2,+\infty)$ is uniformly convex with modules of convexity $\psi$ such that $\psi$ satisfing

$$
\psi \geq 2^{1-p} \min \left\{p 2^{-\frac{p}{2}}, 1-2^{-\frac{p}{2}}\right\}|\cdot|^{p},
$$

Hence, in view of Theorem 2 for function $f(t)=|t|^{p}$ and (2.4), one has

$$
\begin{aligned}
& \left|\frac{a+b}{2}\right|^{p}+\frac{1}{8(b-a)} 2^{1-p} \min \left\{p 2^{-\frac{p}{2}}, 1-2^{-\frac{p}{2}}\right\} \int_{a-b}^{b-a}|t|^{p} d t \\
& \leq\left|\frac{a+b}{2}\right|^{p}+\frac{1}{8(b-a)} \int_{a-b}^{b-a} \psi(t) d t \\
& \leq \frac{1}{b-a} \int_{a}^{b}|t|^{p} d t \\
& \leq \frac{|a|^{p}+|b|^{p}}{2}-\frac{1}{6} \psi(|a-b|) \\
& \leq \frac{|a|^{p}+|b|^{p}}{2}-\frac{1}{6} \min \left\{p 2^{-\frac{p}{2}}, 1-2^{-\frac{p}{2}}\right\}|a-b|^{p} .
\end{aligned}
$$

Proposition 2. Let $p$ be an even number and let $a, b \in \mathbb{R}$ with $0<a<b$, then the following inequality holds:

$$
\begin{aligned}
& (p+1)\left(\frac{a+b}{2}\right)^{p}+\frac{(b-a)^{p+1}}{2^{p+2}(b-a)} \min \left\{p 2^{-\frac{p}{2}}, 1-2^{-\frac{p}{2}}\right\} \\
& \leq \frac{b^{p+1}-a^{p+1}}{b-a} \\
& \leq\left(\frac{a^{p}+b^{p}}{2}-\frac{(b-a)^{p}}{6} \min \left\{p 2^{-\frac{p}{2}}, 1-2^{-\frac{p}{2}}\right\}\right)(p+1) .
\end{aligned}
$$

Proof. The proof is immediate consequence of Theorem 7.

\subsection{Hermite-Hadamard's inequalities for fractional integrals}

Theorem 8. Let $f:[a, b] \rightarrow \mathbb{R}$ be a uniformly convex function. Then, for $\alpha>0$ the following inquality for fractional integrals holds:

$$
f\left(\frac{a+b}{2}\right)+\frac{\Gamma(\alpha+1)}{2^{\alpha+2}(b-a)^{\alpha}} J_{(a-b)^{+}}^{\alpha} \psi(|a-b|) \leq \frac{\Gamma(\alpha+1)}{2(b-a)^{\alpha}}\left[J_{a^{+}}^{\alpha} f(b)+J_{b^{-}}^{\alpha} f(a)\right]
$$




$$
\leq \frac{f(a)+f(b)}{2}-\alpha \beta(\alpha+1,2) \psi(|a-b|) .
$$

Proof. In (1.1), set $t=\frac{1}{2}$, then we have

$$
f\left(\frac{x+y}{2}\right)+\frac{1}{4} \psi(|x-y|) \leq \frac{f(x)+f(y)}{2} .
$$

Now, set $x=t a+(1-t) b$ and $y=(1-t) a+t b$ in (2.5). Multiplying both sides of (2.5) by $t^{\alpha-1}$ and then integrating the resulting inequality with respect to $t$ over $[0,1]$, we obtain

$$
\begin{aligned}
& \int_{0}^{1} t^{\alpha-1} f\left(\frac{a+b}{2}\right) d t+\frac{1}{4} \int_{0}^{1} t^{\alpha-1} \psi(|(2 t-1)(a-b)|) d t \\
& \leq \frac{1}{2} \int_{0}^{1} t^{\alpha-1} f(t a+(1-t) b) d t+\frac{1}{2} \int_{0}^{1} t^{\alpha-1} f((1-t) a+t b) d t .
\end{aligned}
$$

Let $t a+(1-t) b=r,(1-t) a+t b=s$ and $(2 t-1)(a-b)=x$, then

$$
\begin{aligned}
& \frac{f\left(\frac{a+b}{2}\right)}{\alpha}+\frac{1}{4} \int_{b-a}^{a-b}\left(\frac{b-a-x}{2(b-a)}\right)^{\alpha-1} \psi(|x|) \frac{d x}{2(a-b)} \leq \\
& \frac{1}{2} \int_{b}^{a}\left(\frac{b-r}{b-a}\right)^{\alpha-1} f(r) \frac{d r}{a-b}+\frac{1}{2} \int_{a}^{b}\left(\frac{s-a}{b-a}\right)^{\alpha-1} f(s) \frac{d s}{b-a} .
\end{aligned}
$$

So, we have

$$
\frac{f\left(\frac{a+b}{2}\right)}{\alpha}+\frac{1}{2^{\alpha+2}(b-a)^{\alpha}} J_{(a-b)^{+}}^{\alpha} \psi(|a-b|) \leq \frac{\Gamma(\alpha)}{2(b-a)^{\alpha}}\left[J_{a^{+}}^{\alpha} f(b)+J_{b^{-}}^{\alpha} f(a)\right] .
$$

Conversely, since $f$ is uniformly convex one has

$$
f(t x+(1-t) y)+t(1-t) \psi(|x-y|) \leq t f(x)+(1-t) f(y) .
$$

Now, replacing $x$ by $y$ we have

$$
f(t y+(1-t) x)+t(1-t) \psi(|x-y|) \leq t f(y)+(1-t) f(x) .
$$

Adding the two equations (2.6) and (2.7) we obtain

$$
f(t x+(1-t) y)+f((1-t) x+t y)+2 t(1-t) \psi(|x-y|) \leq f(x)+f(y) .
$$

Set $x=a$ and $y=b$ in (2.8) and also multiplying both sides of (2.8) by $t^{\alpha-1}$ and then integrating the resulting inequality with respect to $t$ over $[0,1]$, we obtain

$$
\begin{array}{r}
\int_{0}^{1} t^{\alpha-1} f(t a+(1-t) b) d t+\int_{0}^{1} t^{\alpha-1} f((1-t) a+t b) d t+\int_{0}^{1} 2 t^{\alpha}(1-t) \psi(|a-b|) d t \\
\leq \int_{0}^{1} t^{\alpha-1} f(a) d t+\int_{0}^{1} t^{\alpha-1} f(b) d t
\end{array}
$$

So,

$$
\frac{\Gamma(\alpha)}{2(b-a)^{\alpha}}\left[J_{a^{+}}^{\alpha} f(b)+J_{b^{-}}^{\alpha} f(a)\right] \leq \frac{f(a)+f(b)}{2 \alpha}-\beta(\alpha+1,2) \psi(|a-b|),
$$


which completes the proof.

\section{Applications to SPECial MEANS}

Consider the following special means for two nonnegative real numbers $\alpha, \beta$ with $\alpha \neq \beta$ as follows (see $[3,5,9,10]$ ):

(1) The arithmetic mean:

$$
A=A(\alpha, \beta)=\frac{\alpha+\beta}{2}, \alpha, \beta \in \mathbb{R},
$$

with $\alpha, \beta>0$.

(2) The logarithmic mean:

$$
\bar{L}=\bar{L}(\alpha, \beta)=\frac{\beta-\alpha}{\ln \beta-\ln \alpha}, \alpha \neq \beta, \alpha, \beta \in \mathbb{R},
$$

with $\alpha, \beta>0$.

(3) The generalized logarithmic mean:

$$
L_{n}(\alpha, \beta)=\left[\frac{\beta^{n+1}-\alpha^{n+1}}{(n+1)(\beta-\alpha)}\right]^{\frac{1}{n}}, n \in \mathbb{R} \backslash\{-1,0\}, \alpha \neq \beta, \alpha, \beta \in \mathbb{R},
$$

with $\alpha, \beta>0$.

Proposition 3. Let $a, b \in \mathbb{R}$ with $0<a<b$ and let $p$ be an even number. Then the following inequality holds:

$$
\begin{aligned}
& \left(\left(\frac{a+b}{2}\right)^{p}+\frac{(b-a)^{p+1}}{2^{p+2}(p+1)(b-a)} \min \left\{p 2^{-\frac{p}{2}}, 1-2^{-\frac{p}{2}}\right\}\right)^{\frac{1}{p}} \\
& \quad \leq L_{p}(a, b) \leq\left(\left(\frac{a^{p}+b^{p}}{2}-\frac{(b-a)^{p}}{6} \min \left\{p 2^{-\frac{p}{2}}, 1-2^{-\frac{p}{2}}\right\}\right)\right)^{\frac{1}{p}}
\end{aligned}
$$

Proof. Since the function $g(t)=t^{\frac{1}{p}}$ is increasing for $t \geq 0$ and $p>0$, in view of Proposition 2, the proof is complete.

\section{REFERENCES}

[1] T. Ali, M. A. Khan, and Y. Khurshidi, "Hermite-Hadamard inequality for fractional integrals via $\eta$-convex functions," Acta Mathematica Universitatis Comenianae, vol. 86, no. 1, pp. 153-164, 2017.

[2] H. H. Bauschke, P. L. Combettes et al., Convex analysis and monotone operator theory in Hilbert spaces. Springer-Verlag, 2011, vol. 408.

[3] S. S. Dragomir and R. P. Agarwal, "Two inequalities for differentiable mappings and applications to special means of real numbers and to trapezoidal formula," Applied Mathematics Letters, vol. 11, no. 5, pp. 91-95, 1998, doi: 10.1016/S0893-9659(98)00086-X.

[4] A. Házy and Z. Páles, "On approximately t-convex functions," Publicationes Mathematicae Debrecen, vol. 66, no. 3-4, pp. 489-501, 2005. 
[5] U. S. Kirmaci and M. E. Özdemir, "On some inequalities for differentiable mappings and applications to special means of real numbers and to midpoint formula," Applied Mathematics and Computation, vol. 153, no. 2, pp. 361-368, 2004, doi: 10.1016/S0096-3003(02)00657-4.

[6] J. Makó and Z. Páles, "Implications between approximate convexity properties and approximate Hermite-Hadamard inequalities," Open Mathematics, vol. 10, no. 3, pp. 1017-1041, 2012, doi: 10.2478/s11533-012-0027-5.

[7] J. Makó and Z. Páles, "Approximate Hermite-Hadamard type inequalities for approximately convex functions," Mathematical Inequalities and Applications, vol. 2, pp. 507-522, 2012, doi: 10.7153/mia-16-37.

[8] H. Mohebi and H. Barsam, "Some results on abstract convexity of functions," Mathematica Slovaca, vol. 68, no. 5, pp. 1001-1008, 2018, doi: 10.1515/ms-2017-0162.

[9] C. P. Niculescu, "Convexity according to the geometric mean," Mathematical Inequalities and Applications, vol. 3, no. 2, pp. 155-167, 2000, doi: 10.7153/mia-03-19.

[10] C. P. Niculescu, "Convexity according to means," Mathematical Inequalities and Applications, vol. 6, pp. 571-580, 2003.

[11] M. E. Özdemir, M. Avc1, and E. Set, "On some inequalities of Hermite-Hadamard type via m-convexity," Applied Mathematics Letters, vol. 23, no. 9, pp. 1065-1070, 2010, doi: 10.1016/j.aml.2010.04.037.

[12] M. Z. Sarikaya, E. Set, H. Yaldiz, and N. Başak, "Hermite-Hadamard's inequalities for fractional integrals and related fractional inequalities," Mathematical and Computer Modelling, vol. 57, no. 9-10, pp. 2403-2407, 2013, doi: 10.1016/j.mcm.2011.12.048.

\section{Authors' addresses}

\section{H. Barsam}

Department of Mathematics, Faculty of Science, University of Jiroft, Jiroft, Iran

E-mail address: hasanbarsam1360@gmail.com, hasanbarsameujiroft.ac.ir

\section{A. R. Sattarzadeh}

Department of Mathematics, Faculty of Sciences and Modern Technologies, Graduate University of Advanced Technology, Kerman, Iran

E-mail address: arsattarzadehegmail.com 